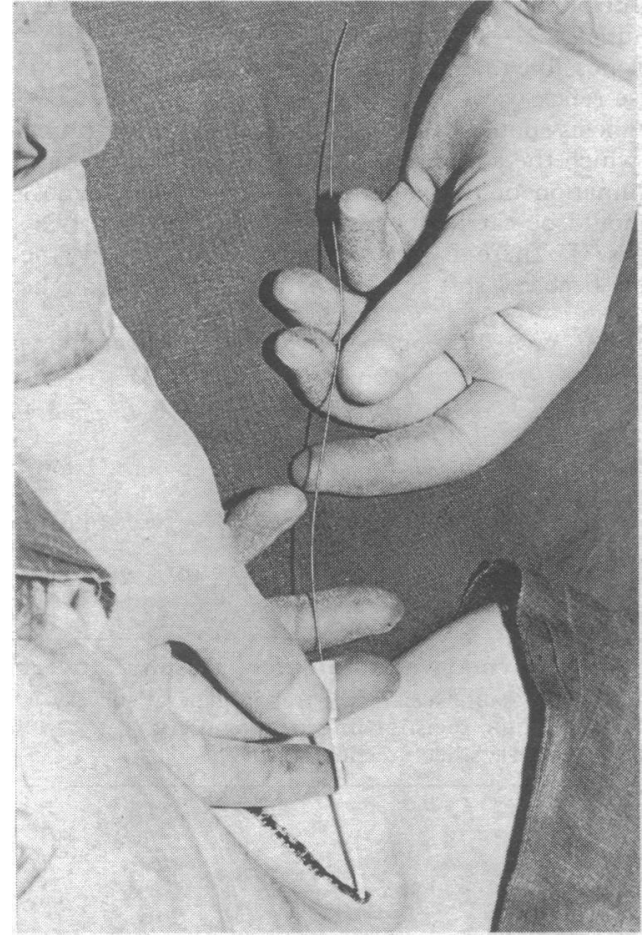

Teflon cannula being passed over floppy-ended guide wire into left subclavian vein by supraclavicular approach.

produce kinking of the cannula and limited the mobility of the patient's neck and shoulder. The guide-wire technique avoids these problems (see illustration). Subsequent nursing care is greatly simplified and provided the usual aseptic precautions are taken there is no increased risk of infection.

${ }^{1}$ Aubaniac, R, Presse Médicale, 1952, 60, 1459

2 Parsa, M H, et al, Bulletin of the New York Academy of Medicine, 1972, 48, 920.

${ }^{3}$ Mogil, R A, et al, Archives of Surgery, 1967, 95, 320.

4 Wilmore, D W, and Dudrick, S J, Archives of Surgery, 1969, 98, 256.

${ }^{5}$ Ellis, B W, and Fielding, L P, fournal of the Royal College of Surgeons of Edinburgh, 1974, 19, 193.

Western General Hospital, Edinburgh EH4 2 XU

JOHN A E GOY, FRACP, MRCP, medical registrar

\section{Raised alpha-fetoprotein levels associated with minor congenital defect}

The value of estimating the level of $\alpha$-fetoprotein $(\alpha-\mathrm{FP})$ in amniotic fluid in diagnosing major defects of the neural tube is well proved. ${ }^{1}$ Amniotic fluid levels of $x$-FP have also been reported to be raised in other serious conditions such as oesophageal atresia and renal tract abnormalities. ${ }^{2}$ A raised $\alpha$-FP level in association with a minor defect, pilonidal sinus, is reported.

\section{Case report}

A 23-year-old woman in her third pregnancy was referred for amniocentesis because her previous child had been born with meningomyelocele and hydrocephalus; he died at the age of 11 weeks. (She had had a spontaneous first-trimester abortion in her first pregnancy.)

A sonar scan confirmed the gestation of 18 weeks with normal outline of fetal head. It also indicated the presence of an ovarian cyst. A laparotomy was performed. The cyst was found to be benign, originating in the left ovary. It was enucleated and the ovary reconstructed. Amniocentesis was performed.
The $x$-FP level was noted to be $120 \mathrm{mg} 1$ (upper limit of normal $30 \mathrm{mg}$ ). A maternal blood sample collected before the operation showed her $x$-FP level to be $40 \mu \mathrm{g} l$ (below the 50th percentile for normal). In view of the high liquor level of $\alpha-F P$, it was decided to repeat the amniocentesis. The level of $x$-FP two weeks later was $130 \mathrm{mg} 1$ (upper limit of normal $20 \mathrm{mg} / 1$ ), and the serum level had risen to $138 \mu \mathrm{g}$ l (95th percentile of normal $=125 \mu \mathrm{g} \mathrm{l}$ ). In view of these results, the patient was effered a termination of the pregnancy, which she accepted. The pregnancy was terminated by extra-amniotic infusion of a solution of prostaglandin $\mathrm{E}$. The fetus had a postanal dimple, which detailed histological examination showed to be a neuroepithelial lined tract with marked vascularity opening on to the skin (seen in photograph). There were no other abnormalities in the fetus.

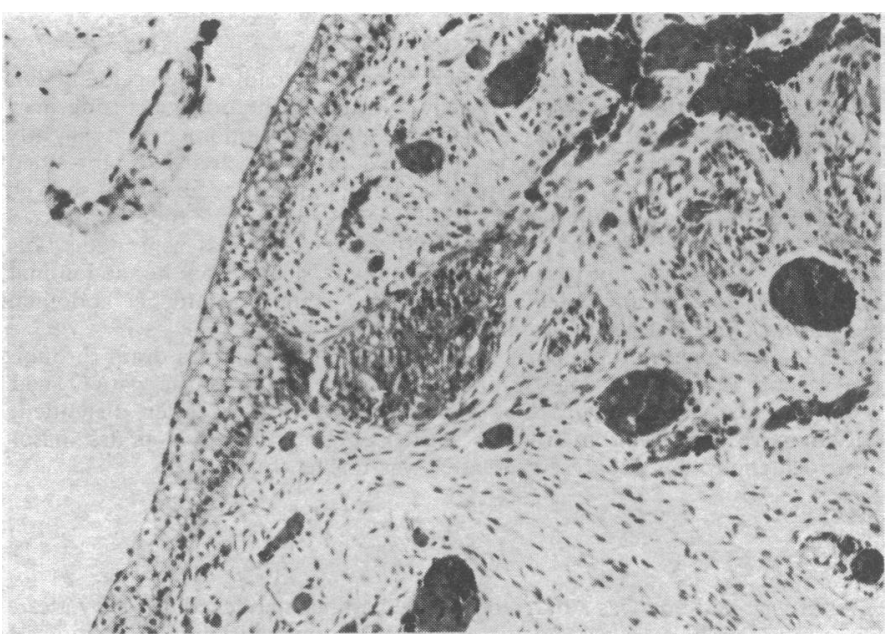

Pilonidal sinus of caudal end showing squamous epithelium with very congested vessels in the vicinity. ( $\because 112$.)

\section{Comment}

All cases of raised $x$-FP levels reported have been associated with neural tube defects or some other gross abnormality of the fetus. The most likely reason for a false-positive diagnosis is contamination of the specimen with fetal blood. A few red blood cells were observed among the cellular material removed from the first liquor specimen for cytogenetic examination, but none were apparent in the second specimen. No haemoglobin $\mathrm{F}$ was detected in the liquor supernatants used for $x$-FP estimations. The high $\alpha$-FP levels obtained in the absence of haemoglobin F were confirmed by Dr J H Brock. The other possibility is that a short spell of fetal hypoxia during enucleation of the ovarian cyst may have resulted in production of an excessive amount of $x$-FP. The amniocentesis performed two weeks later, however, still showed very high levels of $x-F P$.

Pilonidal sinus is a defect that is easily treated and compatible with a normal life. In retrospect termination of the pregnancy was most unfortunate. This case indicates the need for extreme care in interpreting amniotic $x$-FP values and the desirability of obtaining confirmatory evidence of a major neural tube defect before proceeding to termination. Additional investigations that may be helpful include the measurement of other markers such as $x_{2}$-macroglobulin:3 or procedures such as amniography.

We thank Dr D J H Brock, university department of human genetics, Western General Hospital, Edinburgh, for examining both liquor specimens for $x$-FP and haemoglobin F, and Dr John McKenzie, reader in developmental biology, University of Aberdeen, for expert advice. Requests for reprints should be sent to Dr V Jandial, Aberdeen Maternity Hospital, Cornhill Road, Aberdeen.

${ }^{1}$ Brock, D J H, and Sutcliffe, R G, Lancet, 1972, 2, 197.

2 Seppala, M, Obstetrics and Gynaecology, 1973, 42, 613.

3 Brock, D J H, Clinical Genetics, 1975, 8, 297.

4 Connon, A F, Fournal of Obstetrics and Gynaecology of the British Commonwealth, 1974, 8, 753 .

\section{University of Aberdeen, Aberdeen AB9 2ZD}

VIJAY JANDIAL, MB, MRCOG, senior registrar, department of obstetrics and gynaecology

HAZEL THOM, BSC, PHD, senior lecturer, department of child health JANE GIBSON, MB, MRCPATH, lecturer, department of pathology 